\title{
Traceurs isotopiques et glaciologie
}

\author{
PAR
}

\section{Merlivat}

DRA-CEN / Sachay
ET

\author{
C. Lorius \\ Laboratoire de Glaciologie, Grenoble
}

\section{Introduction}

Il a été montré au cours des exposés précédents que l'étude de la distribution des isotopes de l'hydrogène et de l'oxygène dans l'eau constitue une méthodologie nouvelle et complémentaire des méthodes classiques pour l'étude de systèmes hydrologiques.

Nous nous proposons de montrer les conclusions auxquelles peut conduire la mise en auvre de ces mêmes techniques isotopiques lorsqu'elles sont appliquées en glaciologie. Nous nous sommes intéressés pour une part importante à l'étude des calottes polaires Arctiques et Antarctiques et à laquelle seront consacrées les deux premières parties de cet exposé. Dans une troisième partie nous montrerons les résultats d'une étude systématique faite sur un forage de 15 mètres prélevé au Mont Blanc en 1973.

\section{Relations entre teneurs en isotopes stables de la neige et température}

Les isotopes stables de l'hydrogène et de l'oxygence sont dans l'eau sous forme de molécules $\mathrm{HDO}$ et $\mathrm{H}_{2}{ }^{18} \mathrm{O}$, le constituant principal étant $\mathrm{H}_{2}{ }^{16} \mathrm{O}$. Les valeurs des diffusivités dans l'air et des tensions de vapeur saturante de ces trois molécules sont différentes. Lors du refroidissement d'une masse d'air humide, les molécules les plus lourdes se condensent préférentiellement laissant une vapeur résiduelle de plus en plus pauvre en isotopes lourds. La condensation de la vapeur d'eau se faisant à une température d'autant plus basse que son abondance dans le nuage est faible, on observera une relation entre la teneur isotopique des précipitations et la température dans le nuage précipitant. Cette condensation fractionnée des molécules $\mathrm{H}_{2}{ }^{16} \mathrm{O}$, HDO et $\mathrm{H}_{2}{ }^{18} \mathrm{O}$ au cours de la formation des précipitations atmosphériques se traduit par les effets suivants:

1/Variation des teneurs isotopiques des précipitations en fonction de la latitude et de la température moyenne du lieu de précipitation.

Les teneurs isotopiques les plus faibles sont observées aux latitudes les plus élevées.

2/ Variation en fonction de l'altitude

Il y a une corrélation décroissante entre teneur en isotopes et altitude.

3/Variation des concentrations isotopiques suivant les saisons.

Les précipitations hivernales sont plus pauvres en deutérium et oxygène ${ }^{18}$ que les précipitations estivales.

Le premier des 3 points ci-dessus est illustré sur la figure 1 qui représente la teneur moyenne en deuterium des précipitations en différentes stations sur le continent Antarctique en fonction de la température moyenne annuelle dans ces stations [1]. Dans le domaine de température compris entre $-20^{\circ} \mathrm{C}$ et $-35^{\circ} \mathrm{C}$ on observe une relation linéaire entre les deux paramètres d'équation :

$$
\delta \mathrm{D} \% \mathrm{c}=6,04 t\left({ }^{\circ} \mathrm{C}\right)-51
$$

Les mêmes valeurs isotopiques sont représentées sur la figure 2 en fonction de l'altitude des stations, illustrant par là l'effet d'altitude énoncé plus haut.

Les raisons à l'origine du marquage isotopique des précipitations étant identiques pour le deuterium et l'oxygène 18 il existe une relation simple entre les teneurs de ces deux isotopes dans les échantillons. La figure 3 est un diagramme $\delta \mathrm{D}-\delta \mathrm{O}^{18}$ correspondant à une série d'échantillons moyens 


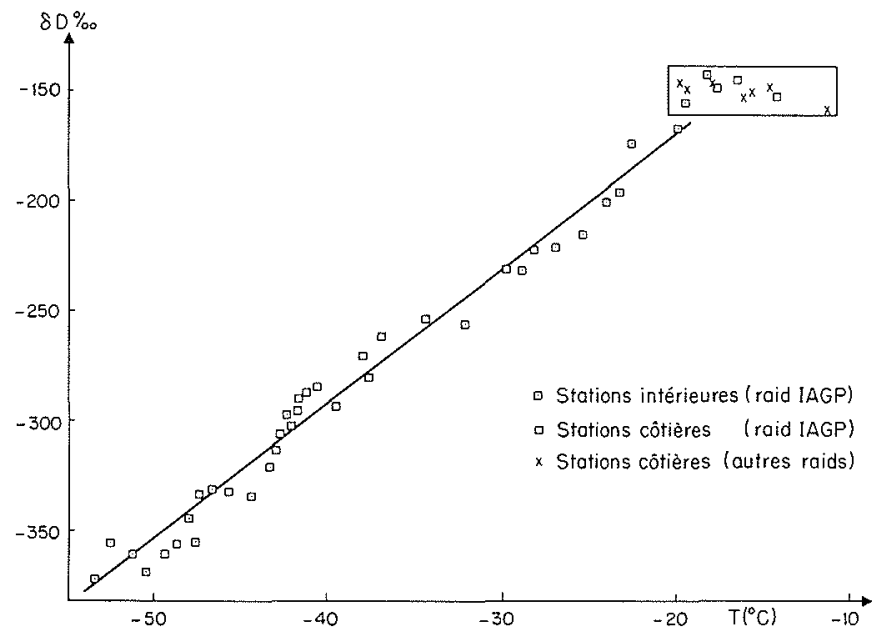

$1 /$ Teneur en deuterium moyenne des précipitations en Antarctique en fonction de la température moyenne annuelle du lieu de précipitation.

I.A.G.P. : International Antarctic Glaciological Program.

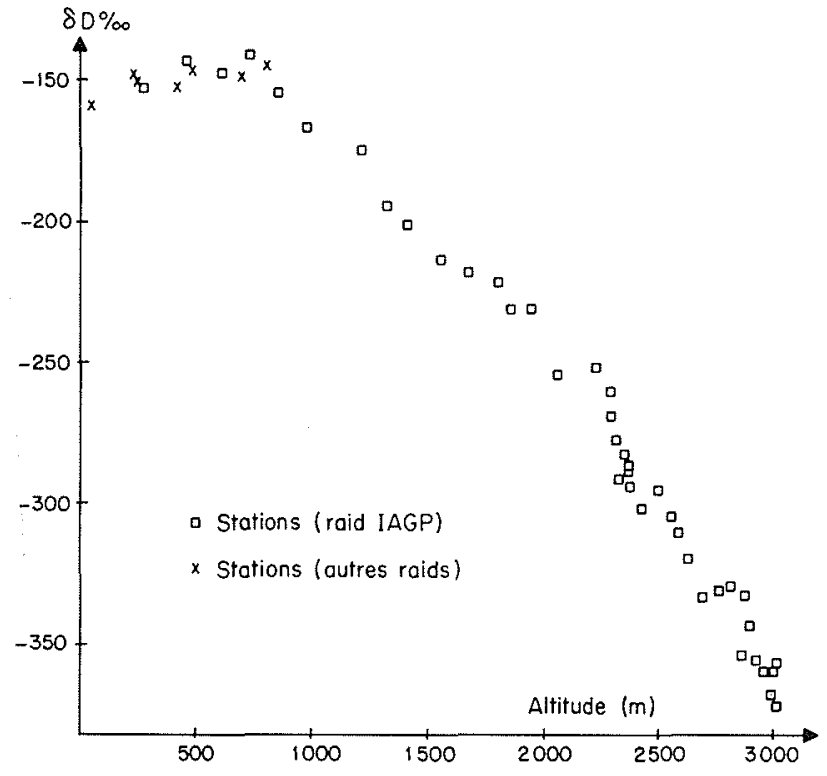

$2 /$ Teneur en deuterium moyenne des précipitations en Antarctique en fonction de l'altitude du lieu de précipitation

de précipitation prélevés aussi en Antarctique [1]. On a tracé la droite représentative des eaux météoriques:

$$
\delta \mathrm{D}=8 \delta \mathrm{O}^{18}+10
$$

Les résultats de la figure 3 illustrent clairement que, en glaciologie, les méthodes isotopiques peuvent être appliquées faisant usage indifféremment des mesures de deuterium ou d'oxygène 18 .

La relation entre la teneur isotopique des échantillons de neige et la température moyenne du lieu de leur précipitation est une empreinte de ce lieu.

Nous montrons sur la figure 4 les résultats obtenus sur les échantillons d'un forage de 300 mètres fait dans la glace de la région côtière de Terre Adélie, pour lequel nous proposons l'interprétation :

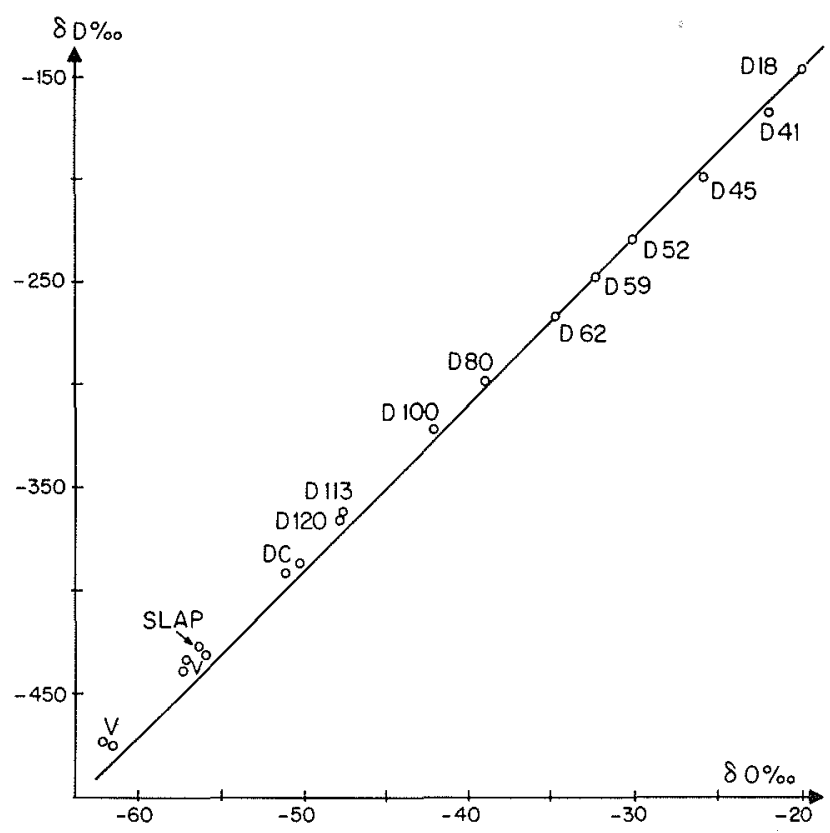

$3 /$ Relation Deuterium-Oxygène 18 dans les échantillons de précipitation.

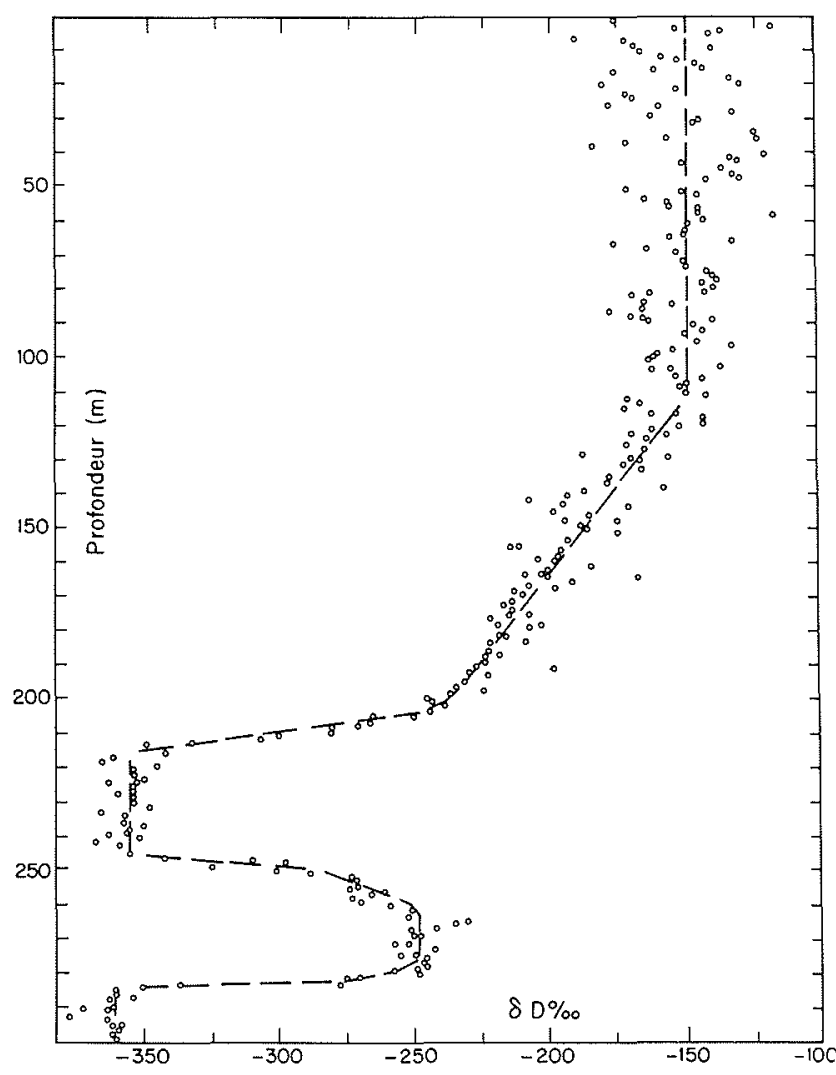

$4 /$ Teneur en deuterium en fonction de la profondeur dans le forage D10 (Antarctique)

- les 110 mètres supérieurs de glace correspondent à des précipitatons d'origine locale,

- la décroissance des teneurs entre 110 et 200 mètres correspond à un écoulement de glace dont l'origine se situe à une distance de l'ordre de $800 \mathrm{~km}$ à l'intérieur du continent, 
- les variations observées dans les 100 derniers mètres montrent un écoulement complexe des tranches de glace lié au relief rocheux au contact du glacier [1].

L'exemple ci-dessus illustre l'intérêt spécifique des techniques isotopiques en glaciologie dans les régions d'écoulement, puisqu'elles permettent d'identifier l'origine des glaces étudiées.

La relation entre température et teneur en isotopes stables a enfin été appliquée à l'étude des paléoclimats. Par analyse des teneurs en oxygène 18 des échantillons's d'un forage profond de 1400 mètres fait dans la glace du Groenland dans une région d'écoulement faible, Dansgaard [2] a pu reconstituer la séquence des évènements climatiques subis par la terre lors des 200000 dernières années.

\section{Répartition du tritium artificiel dans la neige}

Le tritium, isotope radioactif de l'hydrogène de période 12,3 ans a été injecté dans la stratosphère par les explosions thermonucléaires à partir de 1952. Il pénètre dans la troposphère chaque année à des époques privilégiées (au printemps dans chaque hémisphère) à l'état de molécules HTO qui se retrouvent dans les précipitations. En l'absence de fusion, les échantillons d'une carotte de neige ou de glace constitueront un enregistrement de la chronologie de distribution du tritium dans les précipitations, laquelle est fixée par le calendrier des explosions thermonucléaires.

Les figures 5 et 6 montrent la répartition du tritium en fonction de la profondeur dans 2 carottes de neige prises

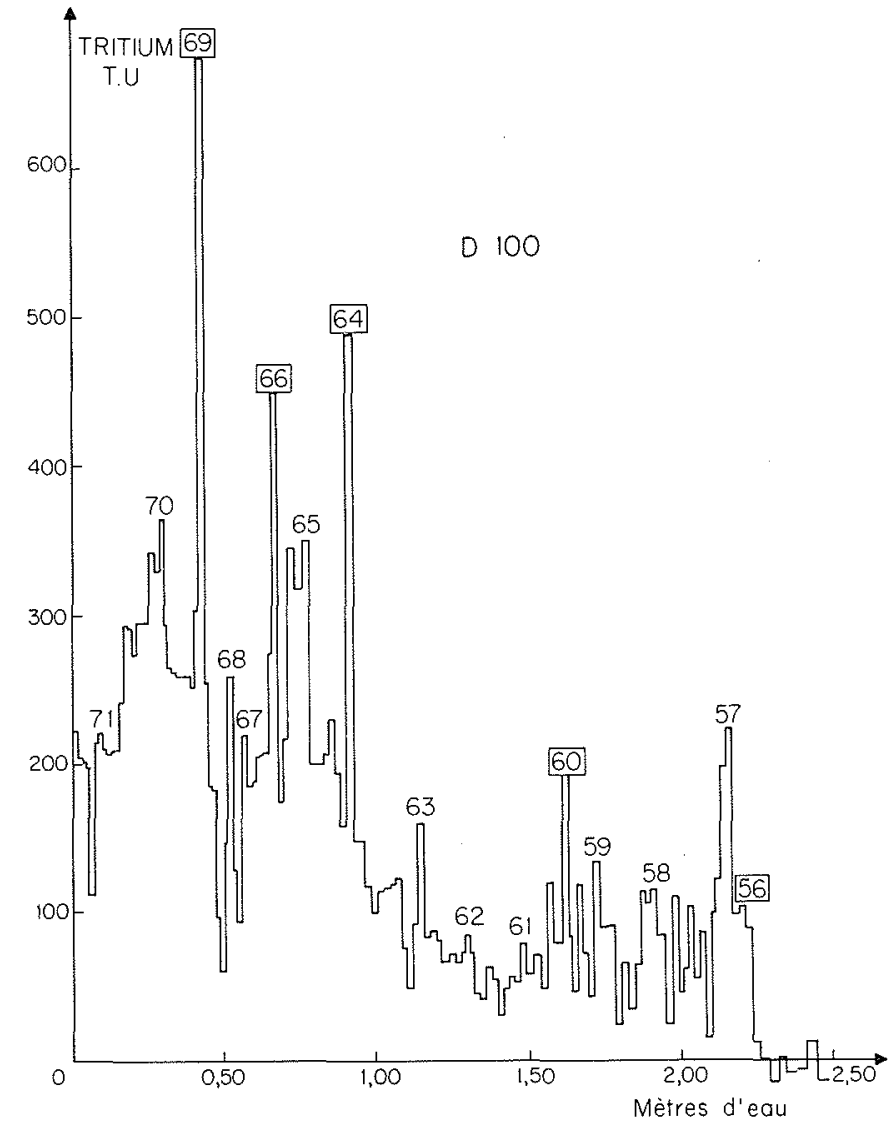

$5 /$ Teneur en tritium en fonction de la profondeur à la station D100 (Antarctique).

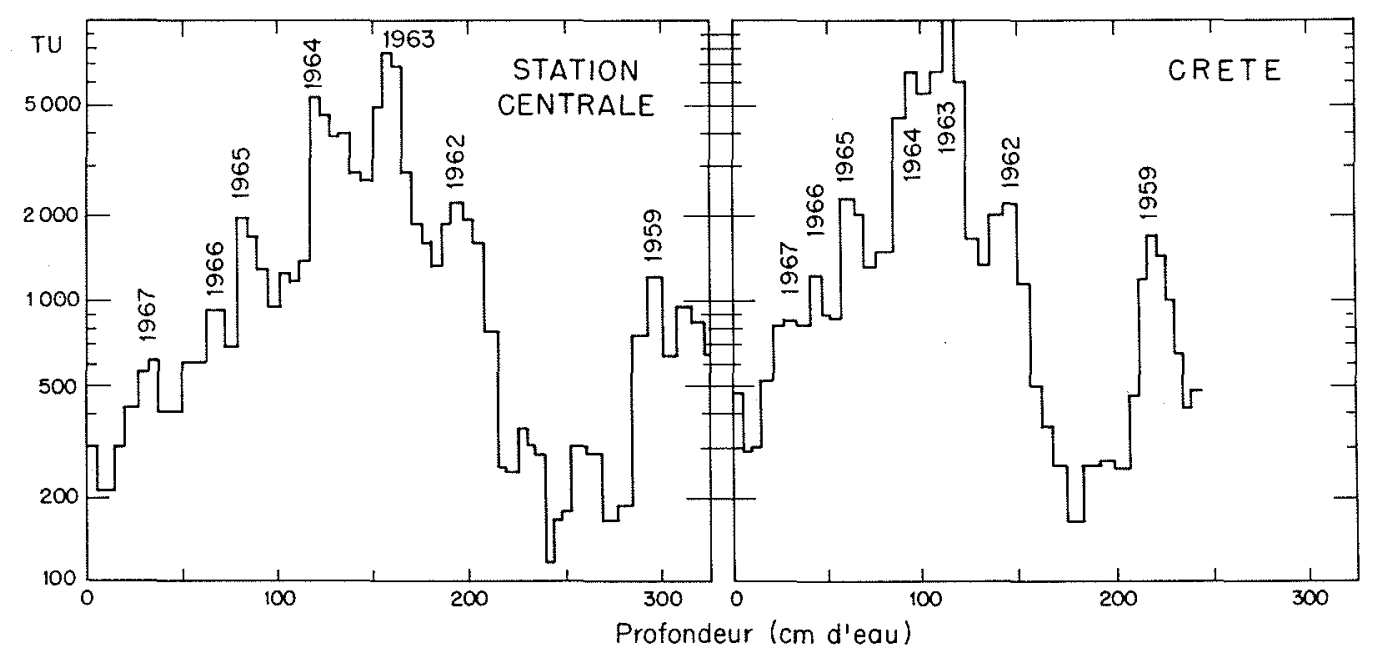

$6 /$ Teneur en tritium en fonction de la profondeur à station centrale et station Crête (Groenland)

l'une au Groenland, l'autre en Antarctique [3, 4]. Les dates ont été attribuées aux différents pics à partir des teneurs mesurées dans les échantillons de précipitation des deux hémisphères. L'existence d'horizons datés permet de connaître avec une assez bonne précision la valeur de l'accumulation de neige en une station donnée. Des études systématiques $[3,4]$ de mesures d'accumulation ont été faites d'une part dans un secteur de l'Antarctique de l'Est compris entre la région côtière de Terre Adélie et une distance de $800 \mathrm{~km}$ à l'intérieur du continent et d'autre part au Groenland dans la région centrale sur un axe ouest-est. Les résultats de cette dernière étude sont représentés sur la figure 7 . La décroissance de l'accumulation moyenne d'eau en fonction de la distance à la côte ouest montre que le régime des précipitations sur la calotte glaciaire est dominé par les vents de secteur ouest. 


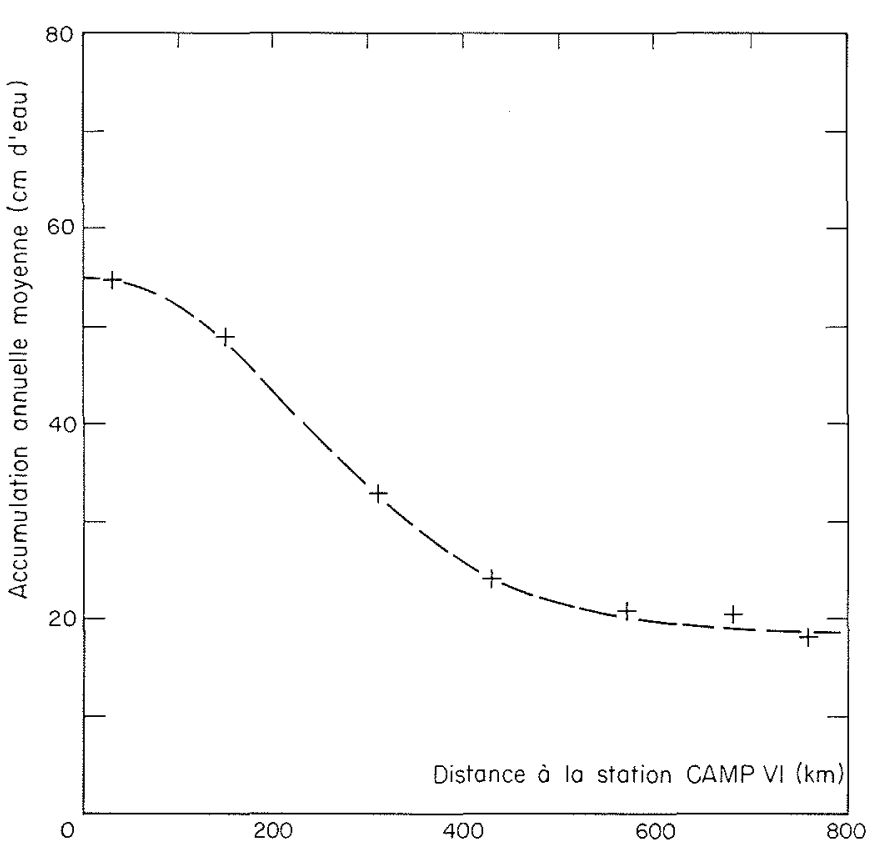

7/ Variation de l'accumulation moyenne annuelle en fonction de la distance à la côte ouest du Groenland

L'exemple ci-dessus a été cité pour illustrer la potentialité des mesures de tritium en glaciologie en fournissant d'une part des informations nécessaires pour l'établissement de bilans de masse en glaciologie (mesures d'accumulation d'eau) et d'autre part des renseignements pour le mécanisme de formation des précipitations.

\section{Etude isotopique d'un forage de neige au Mont Blanc}

Un forage de 15 mètres de profondeur a été fait au sommet du Mont Blanc au cours de l'été 1973 [5]. Cent cinquante échantillons ont été préparés afin d'être analysés par leur teneur en deutérium, tritium et radioactivité artificielle $\beta$. On remarque sur la figure 8 d'importantes fluctuations des teneurs isotopiques, correspondant à des précipitations tombées à des périodes différentes de l'année. La présence de strates de glace à des niveaux correspondant à ces couches de teneur en deutérium élevée permet de repérer les précipitations accumulées au cours des périodes les plus chaudes de l'année. Un premier examen de la figure indique l'existence de 3 cycles isotopiques au moins et peut-être quatre, ce qui conduirait à des valeurs d'accumulations au Mont Blanc de $2,5 \mathrm{~m}$ et $1,9 \mathrm{~m}$ d'eau respectivement. Les mesures de radioactivité artificielle ( $\beta$ global et tritium artificiel) sont actuellement en cours et devront nous permettre de trancher entre ces deux valeurs.

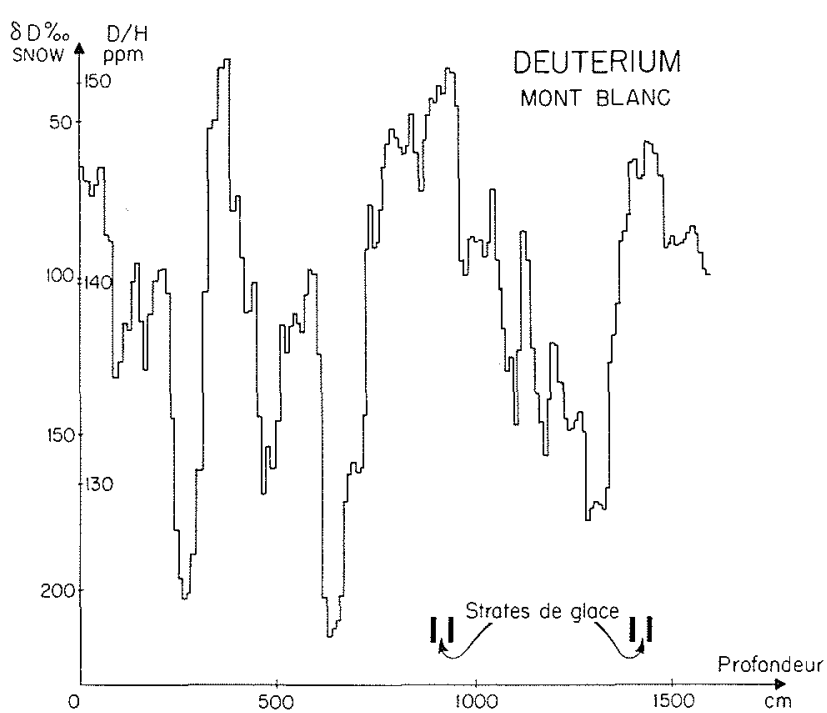

8/Profil en deuterium d'un forage au Mont Blanc

\section{Conclusion}

Nous avons tenté d'exposer les informations que fournissent les isotopes en glaciologie en se servant d'exemples tirés d'études que nous avons faites. Il nous faut toutefois mentionner les applications de cette méthodologie à l'étude des régimes de glaciers tempérés qui sont poursuivies essentiellement en Autriche, en Suisse et au Canada. Les problèmes sont souvent rendus plus complexes dans les régions de moyenne latitude par l'existence de périodes de fusion. Le rôle de mise en archives rempli par une carotte de glace ou de névé n'est plus totalement préservé et en fait on se retrouve confronté avec des problèmes généraux d'hydrologie tels qu'ils ont été présentés dans les exposés précédents.

\section{Bibliographie}

11] LORIUS (C.), MERLIVAT (L.) - Distribution of mean surface stable isotopes values in East Antarctica; Observed changes with depth in coastal area. Présenté à la $16^{\mathrm{C}}$ Assemblée Générale de l'Union de Géodésie et Géophysique Internationale. Grenoble - août 1975. A paraître dans le Journal of Glaciology.

[2] MERlivat (L.), JOUzEl (J.), ROBERT (J.), LORIUS (C.) Distribution of artifical tritium in firn samples from East Antarctica. Présenté à la $16^{\mathrm{e}}$ Assemblée Générale de l'Union de Géodésie et Géophysique Internationale. Grenoble - aoūt 1975. A paraître dans le Journal of Glaciology.

[3] DANSGAARD (W.) et al. - "Climatic record revealed by the Camp Century ice core "in Late Cenozoï ice ages, 37-56, Yale University Press, 1971.

[4] MERLIVAT (L.), RAVOIRE (J.), VERGNAUD (J.P.) and LORIUS (C.) - Tritium and deuterium content of the snow in Groenland Earth and Planetary Science Letters 19, 235-240, 1973.

[5] Pourchet (M.) - Juin 1973. 


\section{Discussion}

Président : M. Jean RODIER

M. Le Président remercie Madame Merlivat pour un exposé qui déborde largement le domaine de la glaciologie et intéressera les climatologues et les hydrologues.

Sur une question de M. Schwertz, Madame MERlivat précise que le graphique donnant la variation avec l'altitude de la teneur en tritium dans les précipitations résulte de mesures effectuées au Groënland entre 1000 et 3000 mètres d'altitude. Des observations faites au Canada par Madame PRANDT donnent des gradients plus faibles. Il est difficile de séparer dans ce phénomène l'effet de l'altitude de l'influence de la continentalité du climat.

Sur ce point, M. Paul-Ch. LEVEQUE apporte les informations suivantes:

"Les "coupes" tritium allant de l'Atlantique à l'Autriche, effectuées par notre laboratoire, ont tout d'abord montré un "effet de continent" bien connu mais aussi un effet d'altitude très net, en Aquitaine, Pyrénées, Massif Central et Centre Aquitaine et en Suisse : Vallée du Rhône et sommets voisins.

M. MARGRITA donne les résultats d'expériences effectuées dans une zone limitée : entre Grenoble, Lux-la-Croix-Haute et le Col de Porte :

"Les mesures de tritium que nous avons réalisées, dit-il, sur des échantillons moyens d'eaux de pluie ont mis en évidence, sans équivoque, un effet de continentabilité. Par contre, des mesures effectuées sur des échantillons mensuels moyens pris localement afin d'éviter les effets de latitude et de longitude - dans la région de Grenoble, par exemple-n'ont pas révélé une influence très nette de l'altitude".

L'hétérogénéité dans ce genre de phénomène est telle, observe $\mathrm{M}$. Le Président, qu'un grand nombre d'expériences est nécessaire pour dégager d'abord une tendance puis aboutir à une conclusion bien nette.

Quand on discute de la répartition des teneurs en tritium dans les précipitations et de l'influence de la "continentalité", remarque $M$. SAUZAY, il faut tenir compte de l'origine des masses d'air génératrices des précipitations et du type de ces demières. Souvent on a utilisé dans ce genre de recherche des teneurs moyennes calculées sur un mois ou quinze jours ; on intègre ainsi dans une même valeur des phénomènes très différents, notamment du fait de la nature des formations nuageuses intéressées.

D'autre part, quelle est l'influence du vent et comment en tenir compte dans l'estimation de l'accumulation glaciaire?

M. MOLINARI évoque un exemple curieux d'utilisation des techniques isotopiques. Il s'agissait d'évaluer, dit-il, les ressources en eau d'une haute vallée andine sur le versant atlantique de l'Argentine, vallée irriguant un vaste bassin agricole dans une zone aride. Nous avons prélevé un échantillon le long de la vallée, d'abord au fil de l'eau, ensuite dans les sources alimentant le cours d'eau et surtout dans la neige couvrant le bassin versant : je précise que ce prélèvement a été effectué en hive entre $1000 \mathrm{~m}$ et $4500 \mathrm{~m}$ d'altitude. L'analyse isotopique a montre d'une façon absolument nette que l'eau s'écoulant dans la rivière n'était pas de "provenance hivernale".

L'intérêt de cette étude a été de montrer qu'avec un échantillonnage sommaire, effectué en un laps de temps très bref, on arrive à obtenir l'information cruciale suivante : durant la période considérée, la neige du haut bassin couvrant des sommets dépassant 6500 mètres d'altitude ne contribue, ni à l'alimentation du ruissellement de surface, ni à celle des nappes ; cette neige est certainement soumise à une ablation éolienne et peut être sublimée, mais elle ne parvient pas jusqu'à la rivière $\left({ }^{1}\right)$.

En l'absence d'autre intervention, M. Le Président clôt la discussion et lève la séance en remerciant toutes les personnes qui ont contribué à la rendre particulièrement instructive.

(1) J. Ch FONTES, I MOLINARI : Reconnaissance isotopique du bassin supérieur du Rio Abaucan (Province de Catamarca, République Argentine). Revue de Géographie Physique et de Géologie dynamique, Paris 1975 (2), Vol. XVII, Fasc. 5 , sous presse). 